\title{
Contribution of the anterior inferior cerebellar artery to cochlear blood flow in guinea pig: A model-based analysis
}

\author{
Tianying Ren ${ }^{\mathrm{a}, \mathrm{b}}$, Alfred L. Nuttall ${ }^{\mathrm{a}}$ and Josef M. Miller ${ }^{*, \mathrm{a}}$ \\ ${ }^{a}$ Kresge Hearing Research Institute, Department of Otolaryngology, The University of Michigan Medical School, 1301 East Ann Street, Ann Arbor, \\ MI 48109-0506, USA; ${ }^{b}$ Department of Otolaryngology, 1st Affiliated Hospital, Xian Medical University, Xian, People's Republic of China
}

(Received 16 March 1993; Revision received 20 July 1993; Accepted 22 July 1993)

\begin{abstract}
This study was performed to determine the contribution of the anterior inferior cerebellar artery (AICA) to cochlear blood flow (CBF) in guinea pig. The AICA and the basilar-vertebral arterial complex in twelve animals was exposed through the basal portion of the skull. The cochlea was ventrally approached and the CBF of the apical area monitored with laser Doppler flowmetry. A specially designed microclamp was held in a micromanipulator and used to obstruct the AICA. When the AICA was clamped, CBF decreased to approximately $60 \%$ of baseline (BL) (not to 'biological zero'), followed by a gradual increase. When the clamp was released, CBF quickly increased to more than $160 \%$ BL and then slowly declined to baseline. To quantify the contribution of AICA to CBF, we formulated an electrical analog model of the cochlear vessel system. With this model, AICA contribution to CBF and the rclationship among blood pressure, blood flow, and vascular resistance or vascular conductance in the cochlea can be explored. Results in the present study indicate that the AICA contributes only about $45 \%$ of CBF to the cochlea; $55 \%$ of $\mathrm{CBF}$ must come from other supplying vessels. Contrary to previous reports, CBF response to AICA clamping did not exhibit a stable or constant decrease but showed time-dependent dynamic changes. In addition, the cochlear vascular system showed a marked autoregulatory response, instead of a passive response, to the perfusion pressure change. AICA clamping is, therefore, not a suitable model for investigation of ischemia effects in the guinea pig cochlea, but it is a useful approach to study autoregulation and the myngenic mechanism of the cochlear vascular system.
\end{abstract}

Microcirculation; Inner ear; Hemodynamics; Autoregulation; Laser Doppler flowmetry

\section{Introduction}

A number of clinical disorders of the inner ear are prcsumcd to reflect a reduced cochlear blood flow (CBF) or an inadequate range of responsiveness of the cochlear vasculature (House, 1975; Short et al., 1985; Miller et al., 1986; Cole and Jahrdoerfer, 1988). Although there have been numerous attempts to define the relationship between impairment of cochlear microcirculation and these disorders, evidence for a vascular etiology is only indirect and appropriate experimental animal models are needed for further investigation in this field. Several authors have obstructed the labyrinthine branches of the anterior inferior cerebellar artery (AICA) to observe hypoxia changes in the guinea pig cochlea (Perlman and Kimura, 1957; Kimura and Perlman, 1958; Perlman et al., 1959; Afzelius and Aursnes, 1979; Short et al., 1985). In order to avoid surgical injury to the auditory nerve and pressure on the brain, Randolf et al. (1990) used microforceps to clamp AICA and induce acute transient local blood flow impairment in the guinea pig cochlea. They re-

\footnotetext{
* Corresponding author. Fax: (313) 764-0014.
}

ported that compression of the cerebellar arteries resulted in variable effects on the laser Doppler flowmeter (LDF) signal from the cochlea. In about half of the experiments, there was a decrease in the laser signal to $25-55 \%$ of the initial level, while in the other cases no clear decrease occurred; complete recovery of CBF after forceps release was not shown. The anterior inferior cerebellar arterial network supplying the rat cochlea has recently been reported by Seidman and Quirk (1992). However, the literature is not clear on how much CBF is contributed by AICA in the guinea pig, an animal that is widely used for cochlear microcirculation studies.

In our previous study (Ren et al., 1992) we found that clamping AICA does not result in a stable or constant decrease but, rather, causes a time-dependent dynamic response in CBF. AICA clamping and release caused very regular and repeatable biphasic exponential changes in CBF. The CBF response curve, with complete recovery, provides a useful approach to investigate the hemodynamics of the cochlear vessels. The purpose of the present study is to use LDF measures of CBF to estimate the percentage of CBF contributed by the AICA in an electrical analog model of the cochlear circulation. 


\section{Methods}

Surgical approach

Twelve healthy young adult guinea pigs weighing $250-420 \mathrm{~g}$ were used in this study. After tranquilization with diazepam $(5 \mathrm{mg} / \mathrm{kg} \mathrm{IP})$, analgesia was provided by fentanyl $(0.32 \mathrm{mg} / \mathrm{kg} \mathrm{IM})$. Anesthesia was maintained by a supplementary half dose of fentanyl every $30 \mathrm{~min}$ and half-dose of diazepam every $2 \mathrm{~h}$. A ventilation tube was inserted into the trachea to insure free breathing. The left or right carotid artery was cannulated and connected to a pressure transducer (P23KL, Statham Instruments, Inc., Oxnard, CA) for continuous blood pressure (BP) and heart rate (HR) measurement with a custom-made electrical circuit. Rectal temperature was maintained at $38 \pm 1^{\circ} \mathrm{C}$ with a servo-regulated heating blanket.

The trachea and esophagus were cut at the level of the fourth tracheal ring, and the superior trachea, the larynx, and the superior esophagus as well as pharynx were elevated upward to expose the surgical field without removing these tissues. Muscular attachments to the skull base were excised and the base of the skull was gently drilled with a diamond bur. The dura was exposed and split, and the arachnoidal membrane covering the basilar and vertebral arteries and their branches was carefully removed.

This study was performed in accordance with the PHS Policy on Humane Care and Use of Laboratory Animals, the NIH Guide for the Care and Use of Laboratory Animals, and the Animal Welfare Act (7 U.S.C. et seq.); the animal use protocol was approved by the Institutional Animal Care and Use Committee of the University of Michigan.

\section{Microclamp and AICA clamping}

A simple microclamp was designed for occluding the AICA (Fig. 1). The tip of a $25 \mathrm{~g}$ hypodermic needle was bent to a $90^{\circ}$ hook and the needle was then attached to a $1 \mathrm{cc}$ plastic syringe barrel from which the plunger had been removed. A $0.25 \mathrm{~mm}$ diameter stainless steel wire, twisted to form a soft spring, was cemented to the end of a camera shutter release cable. The free end of the wire was threaded through the barrel of the syringe and the necdlc so that when the cable tip was seated in the end of the syringe the tip of the wire was positioned about $2 \mathrm{~mm}$ from the angled needle tip. Pressing the cable button advanced the tip of the wire to the hook and it could be secured in this position by tightening the cable set-screw. The entire microclamp system was held in a micromanipulator and positioned so that the AICA was held in the hook. Rapid clamping and release of the vessel were achieved by depressing and releasing the cable button. When the system was in clamp position, the tension from the spring was suffi-

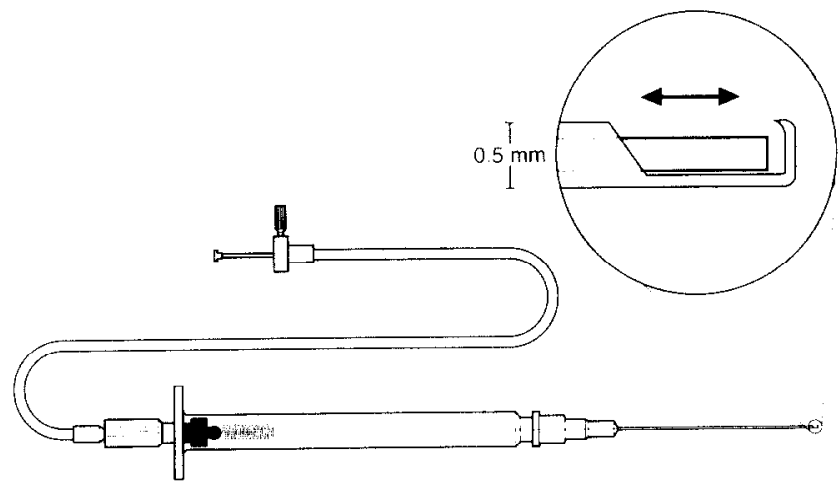

Fig. 1. A diagram of the microclamp. The tip of a hypodermic needle was modified and bent to a $90^{\circ}$ hook and attached to a plastic $1 \mathrm{cc}$ syringe barrel. A $0.25 \mathrm{~mm}$ diameter stainless steel wire was cemented to the end of a camera shutter release cable. Ahout 2 in of the wire was slightly twisted to form a soft spring. The free end of the wire was threaded through the barrel of the syringe and the needle. Pressing the cable button advances the tip of the wire to the hook.

cient to completely occlude the vessel but gentle enough not to damage it even after repeated occlusions.

Although there was great variation in the number, diameter, and site of origin of the cerebellar arteries, the AICA could be easily identified based on one or more of the following characteristics: 1) the largest branch of the basilar artery, between the posterior inferior cerebellar artery (PICA) and the superior cerebellar artery; 2) straight and unbranched inside the surgical ficld; 3 ) clamping causes a significant response in LDF signal. A few small pontine arteries were also found coursing laterally.

\section{Laser Doppler flowmetry}

For LDF, the bulla was exposed using a ventral approach. A $2 \mathrm{~mm}$ diameter hole was made in the bulla, and the cochlear mucosa was gently removed with a small cotton pledget. After the probe was placed on the apex of the cochlea, the hole in the bulla was sealed by removing the soft tissue retractor. CBF was monitored with a Periflux PF2B laser Doppler flowmeter (Perimed Co., Stockholm, Sweden) and PF 303 needle probe. The time constant was set to 0.2 or $0.5 \mathrm{~s}$ and the frequency cutoff to $12 \mathrm{kHz}$. The CBF and BP signals were recorded simultaneously using a chart recorder or a PC computer. In addition, a measure of vascular conductance (VC) of supplying and cochlear vessels was calculated from the ratio of $\mathrm{CBF}$ to $\mathrm{BP}$.

\section{Experimental protocol}

The occlusions were randomly ordered. Each duration was used only once in each animal. The interval between occlusions was long enough to allow complete CBF recovery, i.e., at least $3 \mathrm{~min}$. To observe the effect of longer occlusions, four of the 12 animals received an 
additional 6 min occlusion following the above manipulations.

\section{Estimates of AICA contribution to $C B F$}

During AICA clamping, two mechanisms probably are involved in determining the flow curve: the passive response and the compensatory dilatation or constriction of cochlear vessels to change in perfusion pressure (Ren et al., 1992). These two mechanisms are active simultaneously. Since it is impossible to separate them, we assess the AICA contribution to $\mathrm{CBF}$ with an electric analog model in the current study.

The line segment $A G$ in Fig. 2 indicates the percentage of AICA contribution to CBF. G is determined by the intersection of a vertical line through $A$ and extension of the exponential line $\mathrm{CB}$. $\mathrm{A}$ is the starting point of clamping and $B$ is the minimum $C B F$ during AICA clamping. The AICA contribution to CBF (AG) was expressed as a percent of the initial baseline. Percent AICA contribution of each animal was determined by $30 \mathrm{~s}$ occlusions since shorter occlusions are too short for maximum decrease to be reached.

\section{Statistical analysis}

The mean of values during 1 min immediately before the AICA clamping was used as a baseline (BL) of the LDF measured flow. CBF values were read from the chart recording every $5 \mathrm{~s}$ and expressed as percentage of BL. The data from 6 of 12 animals were collected by computer with an analog to digital recording system. The analog signals of BP and CBF were digi-

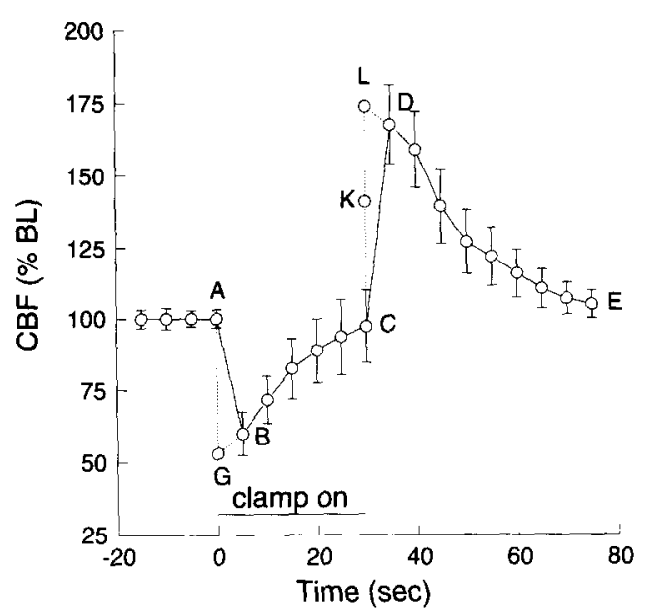

Fig. 2. The graphical determination of the percentage of CBF supplied by the AICA. Each data point and error bar presents the mean and standard deviation $(N=12) . \mathrm{G}$ is determined by the intersection of a vertical line through $\mathrm{A}$ (shown as dashed line here) and extension of the exponential line $\mathrm{CB}$. The percent AICA contribution to $\mathrm{CBF}$ was indicated by $\mathrm{AG}$.

tized at 2 or 4 samples per second. Values presented here are means \pm standard deviations of the percent change from $\mathrm{BL}$ elicited by AICA clamping, unless otherwise stated. The paired $t$ test was used for comparison between the fast decrease amplitude (AG in Fig. 2) and increase amplitude (CL in Fig. 2). Single exponential regression equations were calculated based on $\mathrm{CBF}$ value of slow increase (BC in Fig. 2) and decrease (DE in Fig. 2).

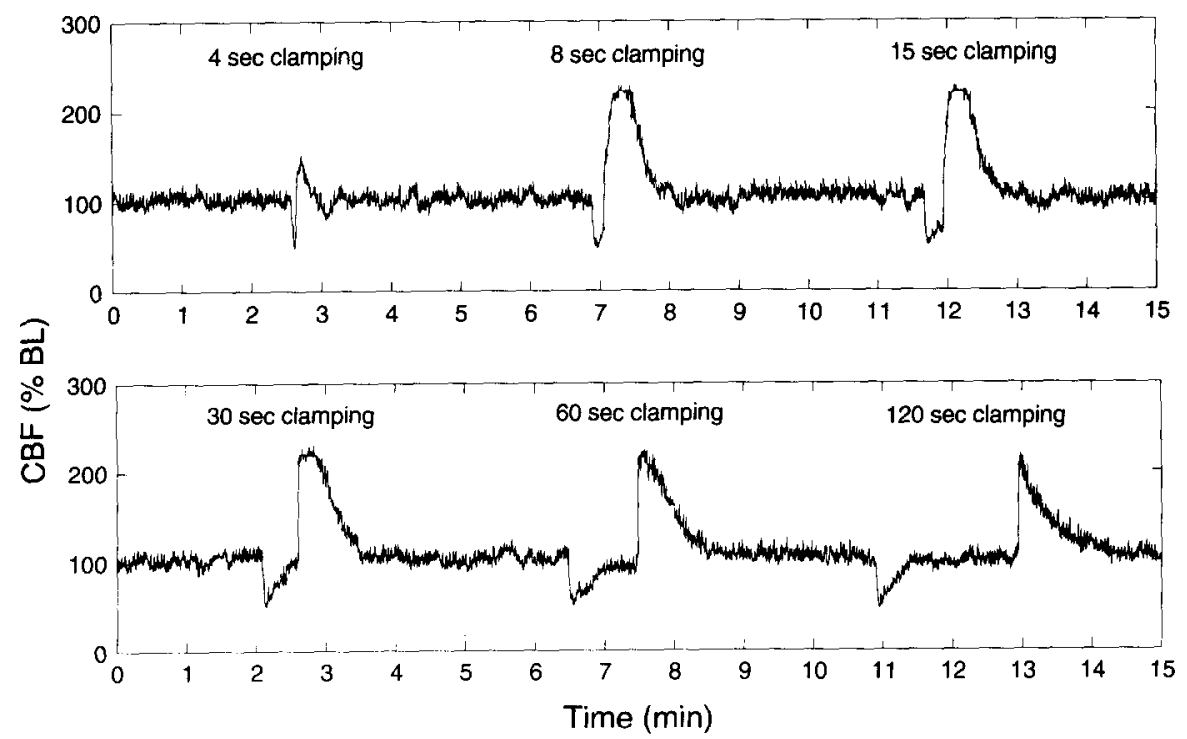

Fig. 3. CBF response to $4,8,15,30,60$, and 120 s AICA clamping in one animal. An abrupt decline in CBF is observed with clamping initiation, followed by a gradual recovery toward the baseline (BL) during the 30,60 , and $120 \mathrm{~s}$ occlusions. Upon clamp release, $\mathrm{CBF}$ increases immediately to more than $160 \% \mathrm{BL}$ within a few seconds and then gradually returns to BL. The response curves for occlusions of shorter duration than $30 \mathrm{~s}$ are quite different in shape and amplitude. 


\section{Results}

All animals tolerated the surgical operation well, and BP was maintained above $41 \mathrm{~mm} \mathrm{Hg}$ throughout all experiments. BP tracing of the computerized recorder showed both regular heart beats and slow irregular oscillations. The means and standard deviation of BP and HR across the animals immediately before occlusions were $50.2 \pm 5.8 \mathrm{mmHg}$ and $327.6 \pm$ 7.8 CPM (cycle per minute). During AICA clamps, no significant change in $\mathrm{BP}$ and $\mathrm{HR}$ was observed. $\mathrm{CBF}$ shows both fast and slow irregular oscillations. The slow irregular fluctuations of CBF were almost always synchronized with BP. All data used in this paper were obtained within $4 \mathrm{~h}$ of anesthesia induction.

\section{$C B F$ response to $A I C A$ clamping}

Fig. 3 shows a series of biphasic $\mathrm{CBF}$ responses to AICA occlusions of different durations in one animal. During the 120,60 , and 30 s occlusions, an abrupt decline in CBF is observed with clamping, followed by a gradual recovery toward BL. Upon clamp release, CBF increased immediately to more than $160 \% \mathrm{BL}$ within a few seconds and then gradually returned to BL. For example, in the 2 min occlusion, CBF immediately decreased to about $60 \% \mathrm{BL}$ (not to the biological zero) in about $4 \mathrm{~s}$ and then gradually increased, reaching the maximum in 30 to $35 \mathrm{~s}$. Note that the rapidly increasing portion of the positive phase is steeper than the initial decreasing portion of the negative phase. Thus, a typical response curve shows a very regular but asymmetric biphasic change. The slowly changing portions have a clear exponential shape. The forms of the responses to 30 and $60 \mathrm{~s}$ occlusions are very similar to that of the 2 min obstruction except for a shorter middle portion. For occlusions of shorter duration than about $30 \mathrm{~s}$, the response curves are quite different in shape and amplitude. This is because CBF did not have time to reach a new 'steady state' level following clamping. For 8 and $15 \mathrm{~s}$ occlusions, the increases immediately after clamp release are slow and the positive peaks are not as sharp as those for 30,60 and $120 \mathrm{~s}$ occlusions. Response to the $4 \mathrm{~s}$ occlusion has a significantly smaller positive peak. These differences probably are related to variable vascular tone in response to different clamping durations.

The CBF responses to $30 \mathrm{~s}$ clamping of 12 AICAs from 12 animals are presented in Fig. 2. The data are exhibited as mean \pm standard deviation. An exponential regression equation for the component labeled $\mathrm{BC}$ was calculated as follows: $\mathrm{CBF}_{\mathrm{t}}=\left(100-45.34 \mathrm{e}^{-0.08 \mathrm{t}}\right) \%$ (BL), $(r=0.967)$, where $t=$ time (seconds) from point $B$ (the range of $t$ is from 0 to $30 \mathrm{~s}$ ), $C B F_{t}=$ value of $\mathrm{CBF}$ at time $\mathrm{t}$. The exponential recovery component following clamp release (DE) is represented by the equation $\mathrm{CBF}_{1}=\left(100+80.51 \mathrm{e}^{-0.04} \mathrm{t}\right) \%(\mathrm{BL}), \quad(\mathrm{r}=$ $0.958)$, where $t=$ time from point $D, C B F_{t}=C B F$ value at time $t$ (the range of $t$ is from 0 to $45 \mathrm{~s}$ ). Because of the short time course of fast decrease (AB) and increase (CD) and the limitations of data acquisition facilities used in this experiment, no data for quantitatively describing $A B$ and $C D$ were available.

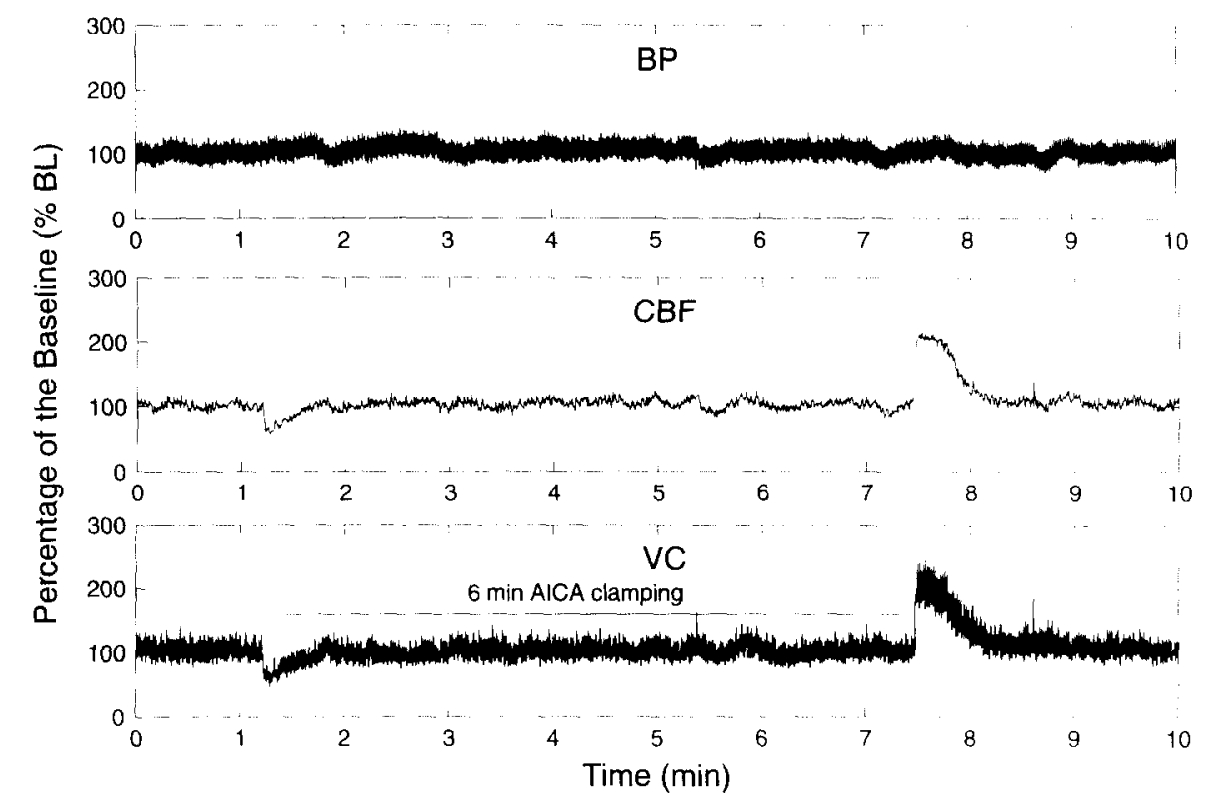

Fig. 4. The relationship among $\mathrm{CBF}, \mathrm{BP}$, and $\mathrm{VC}$. BP did not show significant change during 6 min AICA clamping. CBF decreased temporarily and then returned to the baseline. $\mathrm{CBF}$ increased abruptly after AICA release. Note that there is no significant stable CBF decrease during AICA clamping. VC showed change similar to $\mathrm{CBF}$ but had a more regular biphasic shape, without the slow oscillations caused by BP. 


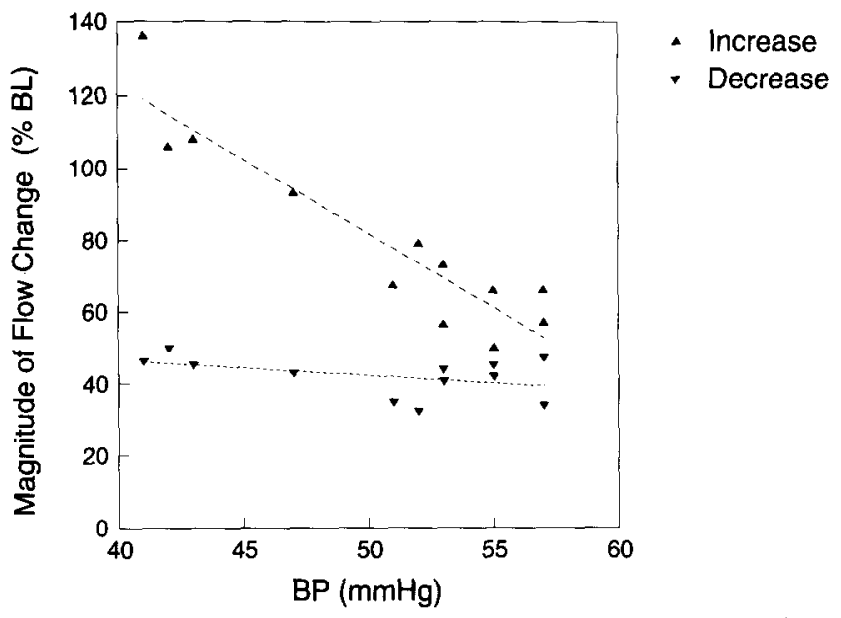

Fig. 5. A negative linear relationship was found between positive peak amplitude and $\mathrm{BP}(\mathrm{y}=299.4-4.3 \mathrm{x}, \mathrm{r}=0.9275, P<0.05)$. No significant relationship existed between fast decrease and BP $(\mathrm{y}=$ 63.3-0.4198x, $\mathrm{r}=-0.4377, P>0.05$ ).

\section{Flow-pressure relationship and vascular conductance}

Relationship among $\mathrm{CBF}, \mathrm{BP}$, and $\mathrm{VC}$ in response to 6 min AICA occlusion is presented in Fig. 4. BP and the heart rate did not show significant change during or after AICA clamping and release. During the clamping time, $\mathrm{CBF}$ does not show a steady decrease but gradually returns to $\mathrm{BL}$ within about $30 \mathrm{~s}$. VC tracing in Fig. 4 shows a response very similar to that of CBF because, according to principles of fluid dynamics, there is a positive linear relationship between flow and hydraulic conductance. However, VC shows a more regular biphasic change pattern, without the slow irregular oscillation caused by BP. Fig. 4 indicates that AICA clamping results in an immediate increase in vascular resistance and a decrease in $\mathrm{VC}$, followed by a gradual return to $\mathrm{BI}$. After clamp release, $\mathrm{VC}$ increases instantly and then gradually returns to $\mathrm{BL}$.

\section{Percentage of AICA contribution to CBF}

The percentage of CBF decrease at $B$ in Fig. 2 $(40.0 \pm 6.2 \% \mathrm{BL}, N=12)$ is significantly smaller than increase at $\mathrm{D}(169.5 \pm 11.5 \% \mathrm{BL}, N=12)(\mathrm{t}=5.755$, $P<0.001)$. Fig. 5 shows a negative linear relationship between $\mathrm{BP}$ and the theoretical positive amplitude (CL in Fig. 2) $(\mathrm{y}=289.7-4.1 \mathrm{x}, \mathrm{r}=0.9275, P<0.05)$, and no relationship was found between the theoretical negative amplitude (AG in Fig. 2) and BP ( $y=63.3-0.4198 x$, $\mathrm{r}=-0.4377, \quad P>0.05)$. The pressure-independent negative amplitude demonstrates that the percentage of AICA contribution to CBF calculated in the current experiment is consistent across the variable BP. The pressure-dependent positive amplitude indicates that the difference between positive and negative phase magnitude (LK in Fig. 2) may reflect pressure-dependency of vascular resistance and conductance.
The mean value of percentage of AICA contribution to $\mathrm{CBF}$ from 12 ears in 12 animals is $45.5 \pm 6.5 \% \mathrm{BL}$.

\section{Discussion}

\section{Electrical analog model}

The non-linear behavior of a vessel is thought to be based on the transmural pressure dependency of the vascular volume and the resistance ( $R$ ) and compliance (C) of the vessel (Spaan et al., 1988). The dynamic CBF response and the amplitude difference between negative and positive phases indicate that volume variation in the cochlear vessel system can be significant and hence make this non-linear approach plausible. A resistance-capacitance network is proposed to interpret the responses of cochlear vessels. In Fig. 6, $P_{\text {in }}$ and $P_{\text {out }}$ are inlet and outlet blood pressure of the cochlear vessel system. $P_{\text {ext }}$ is tissue pressure around the vessels. According to the profile of pressure drops throughout systemic circulation (Gow, 1980), systemic pressure decreases by approximately $90 \%$ at the arteriole and capillary level. Considering the anatomy and physiology of the cochlear vessel system as well as the waterfall phenomenon of blood vessels (Hoffman and Spaan, 1990; Portnoy et al., 1983), it is reasonable to assume that $P_{\text {in }}$ is equivalent to arterial pressure and $P_{\text {out }}$, as well as $P_{\text {cxt }}$, is close to the venous pressure. This assumption is supported by Bohlen et al. (1977) who have shown that, over a wide range of pressures, microvascular pressure in each microvessel is a constant fraction of systemic pressure. In pilot experiments, we found venous pressure in the jugular vein to be 5 to 7 $\mathrm{mmHg}$ and relatively stable. Therefore, the perfusion pressure to the cochlear vessel system is assumed to be close to BP minus the venous pressure.

In the electrical analog model, two parallel resistances, $R_{1}$ and $R_{2}$, are used to represent the supplying vessels. $R_{1}$ is resistance of the AICA and $R_{2}$, resis-

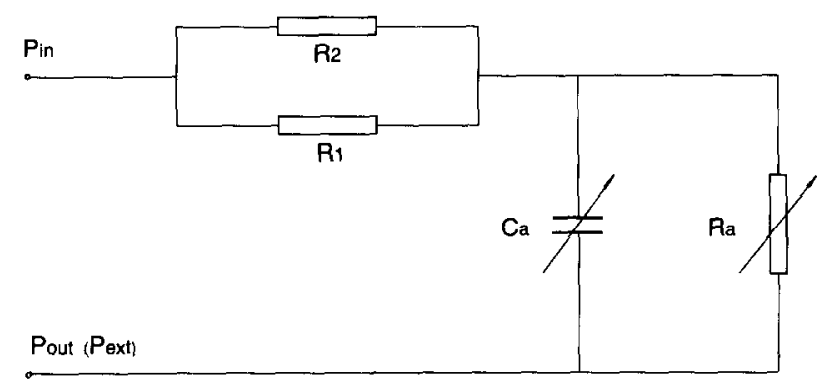

Fig. 6. The proposed elcctrical analog model. $P_{\text {in }}$, the inlet pressure; $\mathrm{P}_{\text {out }}$, the outlet pressure; $\mathrm{P}_{\text {ext }}$, the pressure in tissue around the vessels; $C_{a}$, the compliance of the cochlear vessel system; $R_{1}$, the resistance of the AICA; $R_{2}$, the resistance of the other supplying vessels; $R_{a}$, the resistance of the cochlear vessels. 
tance from the PICA and/or other vessels supplying the cochlear vessels. In this simplified model, $\mathrm{C}_{\mathrm{a}}$ reflects the compliance of the vascular bed, with contributions from both the cochlear vessels and the supplying vessels in series with them. $C_{a}$ is variable because of the pressure-dependency of vascular compliance. Resistance of the cochlear vessels is represented by $\mathrm{R}_{\mathrm{a}}$. Morff and Granger (1982) demonstrated that the autoregulation of blood flow occurs mainly within individual arterioles. Hence, resistances of the large supplying vessels, $R_{1}$ and $R_{2}$, are proposed to be relatively constant and $R_{a}$, contributed mainly by the arterioles, to be variable, depending upon the transmural pressure and reflecting the autoregulatory property of the cochlear vessels.

\section{AICA contribution to $C B F$}

During AICA clamping, CBF did not reach biological zero. Furthermore, the change gradually recovered to the BL value or exhibited an overshoot. This implies a multiple vessel supply to the cochlea. Fast $\mathrm{CBF}$ decrease ( $\mathrm{AB}$ in Fig. 2) is probably determined mainly by passive properties, capacitance $\mathrm{C}_{\mathrm{a}}$ and resistance $\mathrm{R}_{\mathrm{a}}$ immediately before the clamping; gradual increase during the clamping ( $\mathrm{BC}$ in Fig. 2) is controlled by an active behavior, i.e., autoregulation or a myogenic response (Ren et al., 1992). Since at time zero after AICA clamping neither the active nor passive mechanism mentioned above has had time to change, the $\mathrm{CBF}$ was determined only by the change in perfusion pressure caused by the clamping. Thus, AG in Fig. 2 was used to indicate the percentage contribution of CBF. In this way, the contribution of AICA to CBF under physiological conditions can be estimated. If a one-step decrease in perfusion pressure is assumed with AICA clamping, the same scale of one-step increase in perfusion pressure is expected with AICA release. Thus, one would hypothesize that the ratio of CBF change in response to AICA release should be the same as that in response to AICA clamping; the CBF curve would be a symmetrical biphasic response. But the data presented in this paper do not support this hypothesis. We believe the main reason for the asymmetry is the differing compliance at the start and end of AICA clamp. This compliance is related to the nonlinearity of the pressure dependent vessel volume (Gow, 1980). Before AICA clamping vascular compliance is smaller than immediately before AICA release. Thus, the flow response for AICA release is larger than for AICA clamp. That there is no correlation between the calculated AICA contribution to CBF and BP (Fig. 5) supports our suggestion that the measurement based on proposed model is appropriate.

Since it is well known that the AICA provides the arterial blood supply to the membranous labyrinth (Axelsson, 1968; Lawrence, 1980), it is reasonable to occlude the AICA to produce acute transient local hypoxia in the guinea pig cochlea. In the study by Randolf et al. (1990), successive proximal occlusions of the terminal cerebellar arteries resulted in a drop in the cochlear LDF signal to $25-55 \%$ of its initial level in about half of the experiments, while in the other cases no clear decreases occurred. The data obtained in the current experiment show a time-dependent change of $\mathrm{CBF}$ following $\triangle \mathrm{IC} \Lambda$ clamping and release. Little or no stable decrease was found during AICA occlusion. On the basis of the electrical analog model and the dynamic CBF response to AICA clamping and release, the current study indicates that the AICA contributes only about $45 \%$ of CBF in guinea pig. In a pilot study, brief proximal occlusion of the basilar artery caused CBF to decrease approximately $80 \%$ of $\mathrm{BL}$. These results indicate that $\mathrm{CBF}$ derives mainly from the vertebrobasilar vascular system and probably passes through AICA and its terminal anastomoses with PICA, the superior cerebellar artery, and/or small pontine arteries. Pronounced occurrences of anastomoses between the cerebellar arteries have been observed in the cat (Bernstein and Silverstein, 1966; Costa et al., 1966).

Results of the current experiment indicate a multiple source of CBF leading to the labyrinthine artery, suggesting AICA clamping is not a suitable model in studying the effects of cochlear ischemia in guinea pig. Since the measurement approach introduced in this paper can provide more quantitative information on CBF dynamics than other reported techniques, it is an appropriate model for investigating CBF autoregulation and the local effects of neural factors, pharmacological agents, and other vasoactive substances.

\section{Acknowledgments}

Authors gratefully acknowledge editorial help given by Nadine Brown and Kelly Gizowski.

This work was supported by the NIH grant DC 00105 and AG 08885 of U.S.A. and the National Natural Science Foundation of China. The work was done during a WHO fellowship.

\section{References}

Afzelius, L.E. and Aursnes, J. (1979) Structural changes in the organ of Corti of the guinea pig after obstruction of the arterial blood flow to the inner ear. Acta Otolaryngol. (Stockh.) 88, 183-186.

Axelsson, A. (1968) The vascular anatomy of the cochlea in guinea pig and in man. Acta Otolaryngol. (Stockh.) Suppl. 243, 1-134.

Bernstein, J.M. and Silverstein, H. (1966) Anterior cerebellar and labyrinthine arteries. A study in the cat. Arch Otolaryngol. 83, $422-435$. 
Bohlen, J.G., Gore, R.W. and Hutchins, P.M. (1977) Comparison of microvascular pressures in normal and spontaneously hypertensive rats. Microvas. Res. 13, 125-130.

Cole, R.R. and Jahrdoerfer, R.A. (1988) Sudden hearing loss: An update. Am. J. Otol. 9, 211-215.

Costa, O.A., Thalmann, R. and Covell, W.P. (1966) Arterial perfusion of the inner ear. I. The method. Laryngoscope 76, 1874-1888.

Gow, B.S. (1980) Circulatory correlates: Vascular impedance, resistance, and capacity. In: D.F. Bohr, A.P. Somlyo and H.V. Jr. Sparks (Eds.), Handbook of Physiology. Sec. 2: The Cardiovascular System, Vol. 2: Vascular Smooth Muscle, American Physiological. Society, Bethesda, MD, pp. 353-408.

Hoffman, J.I. and Spaan, J.A. (1990) Pressure-flow relations in coronary circulation. Physiol. Rev. 70, 331-390.

House, W. (1975) Meniere's disease. Management and theory. Otolaryngol. Clin. North. Am. 8, 515-535.

Kimura, R. and Perlman, H.B. (1958) Arterial obstruction of the labyrinth. I. Cochlear changes. Ann. Otol. Rhinol. Laryngol. 67, 5-24.

Lawrence, M. (1980) Control mechanisms of inner ear microcirculation. Am. J. Otolaryngol. 1, 324-333.

Miller, J.M., Hultcrantz, E., Short, S.O. and Nuttall, A.L. (1986) Pharmacological effects on cochlear blood flow measured with the laser Doppler technique. Scand. Audiol. Suppl. 26, 11-20.

Morff, R.J. and Granger, H.J. (1982) Autoregulation of blood flow within individual arterioles in the rat cremaster muscle. Circ. Res. $51,43-55$.
Perlman, H.B. and Kimura, R. (1957) Experimental obstruction of venous drainage and arterial supply of the inner ear. Ann. Otol. Rhinol. Laryngol. 66, 537-546.

Perlman, H.B., Kimura, R. and Fernandez, C. (1959) Experiments on temporary obstruction of the internal auditory artery. Laryngoscope 69, 591-613.

Portnoy, H.D., Chopp, M. and Branch, C. (1983) Hydraulic model of myogenic autoregulation and the cerebrovascular bed: The effects of altering systemic arterial pressure. Neurosurg. 13, 482498.

Randolf, H.B., Haupt, H. and Scheibe, F. (1990) Cochlear blood flow following temporary occlusion of the cerebellar arteries. Eur. Oto-Rhi-Laryngol. 247, 226-228.

Ren, T.Y., Avinash, G., Nuttall, A.L., Miller, J.M., Laurikainen, E. and Quirk, W.S. (1992) Hemodynamics of cochlear blood flow in the guinea pig. Abstr. Assoc Res. Otolaryngol. 305.

Seidman, M.D., and Quirk, W.S. (1992) The anterior inferior cerebellar arterial netwok supplying the rat cochelea and its role in autoregulation of cochlear blood blow. Eur. Oto-Rhi-Laryngol. $249,332-335$.

Short, S.O., Goodwin, P.C., Kaplam, J.N. and Miller, J.M. (1985) Measuring cochlea blood flow with laser Doppler spectroscopy. Otolaryngol. Head Neck Surg. 43, 786-793.

Spaan, J.A., Dankelman, J., Kouwenhoven, E., Verburg, J., Bruinsma, P. and Vergroesen, I. (1988) Non-linear system analysis of the coronary circulation. INSERM 183, 54-64. 